Supporting Information for

\title{
A Novel Potent and Highly Specific Inhibitor against Influenza Viral N1-N9 Neuraminidases: Insight into Neuraminidase-inhibitor Interactions
}

\author{
Nongluk Sriwilaijaroen, ${ }^{*}$ Sadagopan Magesh, Akihiro Imamura, Hiromune Ando, Hideharu \\ Ishida, Miho Sakai, Erika Ishitsubo, Takanori Hori, Setsuko Moriya, Takeshi Ishikawa, \\ Kazuo Kuwata, Takato Odagiri, Masato Tashiro, Hiroaki Hiramatsu, Kenji Tsukamoto, Taeko \\ Miyagi, Hiroaki Tokiwa, ${ }^{*}$ Makoto Kiso, ${ }^{*}$ and Yasuo Suzuki
}

\section{Table of Contents}

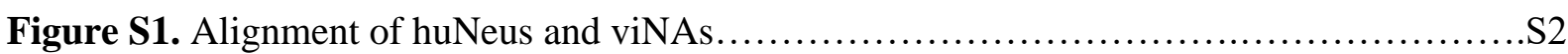

Figure S2. Binding of Pen-GUN in N1-N9 viNA active sites.............................. 5

Figure S3. Binding of Pen-GUN in cartoon diagrams of wild-type compared with H274Y-N1

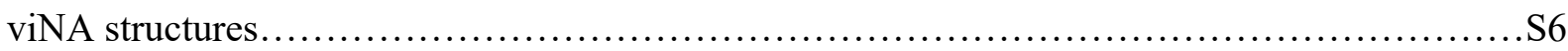

Table S1. Comparison of IFIE values of Pen-GUN to cPro-GUN interactions with each important

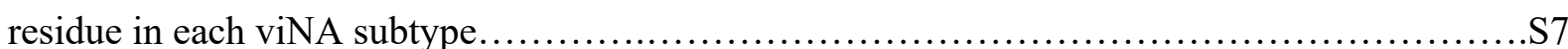

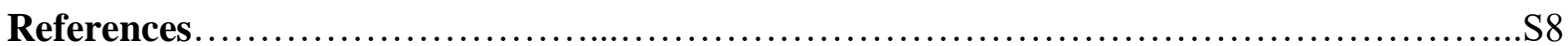




\section{Human neuraminidases}

huNeu1 1 MTGERPSTALPDRRWGPRILGFWGGCRVWVFAAIFLLLSLAASWSKAENDFGLVQPLVTMEQLLWVSGRQIGSVDTFRTPLITATPRG-TLLAFAEARKM

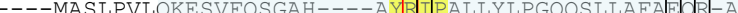
huNeu3 1 ---MRP-ADLP-PRP------------MEESPASSSAPTETEEPGSSAEVMEEVTTCSFNSPLFRQEDDRGITYAIPALLYIPPTHTFLAFAE ER-S

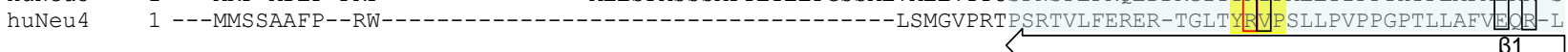

huNeul 100 SSSDEGAKFIALRRSMDQG----STWSPTAFIVNDGDVPDGINIGAVVSDVETGVVFLFYFS耳CAHK-AGCQVASTM------LVWSKDDGVSWSTPRNL huNeu2 43 SKKDEHAELIVLRRGDYDAPTHQVQWQAQEVVAQARLDGHRSMNPCPLYDAQTGTLFLFF IAI PGQVI EQQQLQTRANVTRLCQVTSTDHGRTWSSPRDL huNeu3 47 TRRDEDALHLVLRRGLRIG--QLVQWGPLKPLMEATLPGHRTMNPCPVWEQKSGCVFLFFIOVRGHVERQQIVSGRNAARLCFIYSQDAGCSWSEVRDL huNeu4 45 SPDDSHAHRLVLRRGTLAG--GSVRWGALHVLGTAALAEHRSMNPCPVHDAGTGTVFLFELAULGHTPEAVQIATGRNAARLCCVASRDAGLSWGSARDL

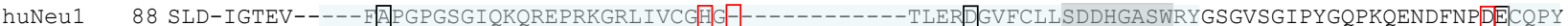
huNeu2 143 TDAAIGPAYREWSTHA VGPGHCLQL--HDRARSLVVPAYAYRKL------HPIQRPIP SAFCFLSHDHGRTWARGHFVA-----------QD-TLECQVA huNeu3 145 TEEVIGSELKHWAT A AGPGHGIQL----QSGRLVIPAYTYYIPSWFFCFQLPCKTR PHSLMIYSDDLGVTWHHGRLIR-----------PMVTVECEVA huNeu4 143 TEEAIGGAVQDWATEAVGPGHGVQL----PSGRLLVPAYTYRVD-RRECFGKICRTSEHSFAFYSDDHGRTWRCGGLVP-----------NLRSGECQLA

huNeu1 269 ELPDGS----VVINARNQNNYHCHCRIVLRSYDACDTLRPRDVTFDP-----ELVD-PVVAAG------------------------------------

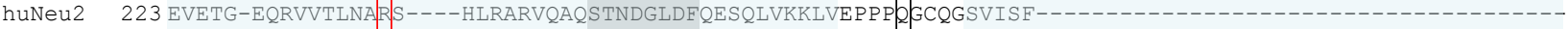
huNeu3 230 EVTGRAGHPVLYCSART----PNRCRAEALSTDHGEGFORLALSRQLCEP-PHGCQGSVVSFRPLEIPHRCODSSS----------------------huNeu4 227 AVDGGQAGSFLYCNARS----PLGSRVQALSTDEGTSELPAERVASLPET-A]GCQGSIVGF-PAPAPNRPRDDSWSVGPGSPLQPPLLGPGVHEPPEEA A

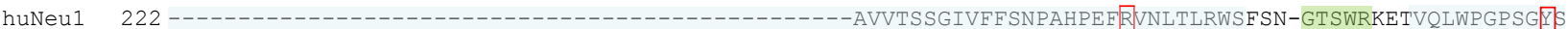

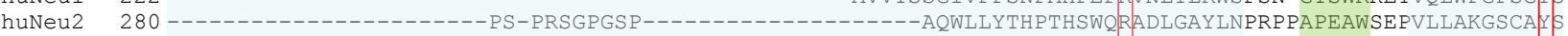

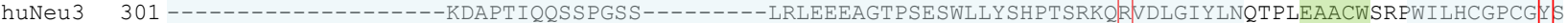

321 AVDPRGGQVPGGPFSRLOPRGDGPROPGPRPGVSGDVGSWTLALPMPFAAPPOSPTWLIYSHPVGRRARTHMGTRTSOSPIDPRSWTEPWTIYEGPSGYS $\beta 5$

huNeu1 372 SLATLEGSMDGEEQAPQLYVLYEKGRNHYTESISVAKISVYGTL--------------------

huNeu2 336 DLOSMGTGPDGS---PLFGCLYEAN---DYEEIVFLMFTLKOAFP---AEYLPQ------------

huNeu3 372 DLAALE--EEG-----LFGCLFEGTKQECEQIAFRLFTHREILSHLQGDCTSP--GRNPSQFKSN-

huNeu4 421 DLASIGPAPEGG---LVFACLYESGARTSYDEISFCTFSLREVLENVPASPKPPNLGDKPRGCCWPS

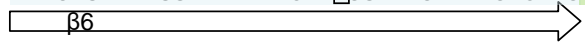




\section{Influenza virus neuraminidases}

dkN1/ 05

mlN1/ 09

$\mathrm{dkN} 2 / 05$

$\mathrm{dkN} \mathbf{N} / 05$

dkN4 / 07

dkN5/06

$\mathrm{dkN} 6 / 04$

dkN7 / 07

$\mathrm{dkN} \mathbf{8} / 06$

dkN9/ 05

CApdmN1/ 09

NApdmN1/09

YAhuN1/06

KIhuN1/06

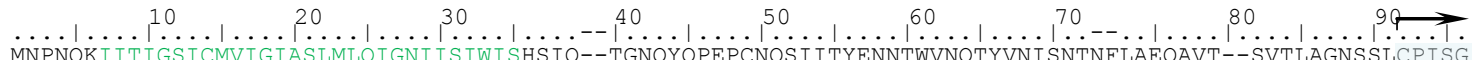
MNPNQKIITIGSICMVIGIASLMLQIGNMISIWVSHSIQ--TGNQYQPEPCNQSI ITYENNTWVNQTYVNISNTNFLTEQAVT--SVALAGNSSLCPISG MNPNQKIITIGSVSLTIATVCFLMQIAILATTVTLHFKQ--NECSIPSNNQVVPCEPI I IERNITEIVYLNNTT--IEKEICPEVLEYRNWSKPQCQITG MNPNQKIITIGVANTTLSTIALLIGIGNLIFNTVIHEKI--GDHQTVVYPTIT--APVVPNCSDTIITYNNTVVNNITTTIITKAEKHFKSSLPLCPERG MNPNOKIITIGSVS IVLTTI GLILOITSLCSIWFSHYNO--VT-OTSEOPCSNNTTNYYNETFVNVTNVONNYTTITEPSSPO--I I HYSSGKDLCPVRG MNPNQKIITIGS ISLGLVVFNILLHVAS IVLGIIS------VTKDHEAYTCNTTEV--YNETVRVKTVI I PVNNTIYIERELTHEPKFLNNTEPLCEVSG MNPNQKI ICISATGMTLSVVSLLIGIANLGLNIGLHYKVGDTPDVNI PNMNGTNSTTTI INN-HTQNNFTNITN-I IVNKNEKGT--FLNLTKPLCEVSS MNPNOKLFALSGVAIALSVLNLLIGISNVGLNVSLHLKE----KGPKQEENSTCTTINQNNTTVVENTYVNNTT-I ISKETELKTPSYLLLNKSLCNVEG MNPNQKIITIGSISLGLVVFNVLLHVVSI IVTVLV------LGRGGNNGICNETVVREYNETVRIEKITQWHNTSVVEYVPYWNEGTYMNNTEAICDVKG MNPNQKILCTSATAIVIGTIAVLIGVANLGLNI GLHLKP--SCNCSHSQPEATNTSQT I INNYYNETNITQISN-TNIQMEERASRGFNNLTKGLCT INS MNPNQKIITIGSVCMTIGMANLILQIGNIISIWISHSIQ--LGNQNQIETCNQSVITYENNTWVNQTYVNISNTNFAAGQSVV--SVKLAGNSSLCPVSG MNPNOKIITIGSVCMTIGMANLILOIGNIISIWISHSIQ--LGNONQIETCNOSVITYENNTWVNQTYVNISNTNFAAGQSVV--SVKLAGNSSLCPVSG MNPNQKIITIGSISIAIGIISLILQIGNIISIWASHSIQ--TGSQNHTGICNQRIITYENSTWVNNTYVNINNTNVVAEKDKT--SVTLAGNSSLCSISG MNPNQKIITIGSISIAIGI ISLILQIGNI ISIWASHSIQ--TGSQNHTGICNQRI ITYENSTWVNNTYVNINNTNVVAEKDKT--SVTLAGNSSLCSISG
$\mathrm{dkN} 1 / 05$

$\mathrm{mlN} 1 / 09$

$\mathrm{dkN} \mathbf{2} / 05$

dkN3/05

$\mathrm{dkN} \mathbf{4} / 07$

$\mathrm{dkN} 5 / 06$

dkN6/04

$\mathrm{dkN} \mathbf{7} / 07$

$\mathrm{dkN} \mathbf{8} / 06$

dkN9/05

CApdmN1/09

NApdmN1/ 09

YAhuN1/06

KIhuN1/06 $\ldots 100 \ldots|\ldots| \ldots|\ldots| \ldots$ WAIYSKDNGIRIGSKGDVFVIR FAPFSKDNSIRLSAGGDIWV' FFPFHKDNAIRLGENKDVIV WAPLSKDNGIRIGSRGEVFV FAIVSKDNGIRIGSRGHVFVI WHILSKDNAIRIGEDAHILVT WVVIAKDNAVRFGESEQI IV FAPFSKDNGIRIGSRGHVFVI WHIYGKDNAVRIGENSDVLVI WAIYSKDNSVRIGSKGDVFVI TAIYSKDNSVRIGSKGDVFV WAIYTKDNSIRIGSKGDVFV $\mathrm{C}^{\text {WAIYTKDNSIRIGSKGDVF }}$

200
120

130 140 150 160 170 180 190

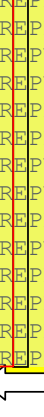
FISCSHLECRTFFLTQGALLNDK ISCSHLECRTFHLTQGALLND YVSCDNDDCWSFALAOGALLGTK ISCS INECRTFFLTQGALLNDK FVSGPSECRTFFLTQGALLNDK YLSCDPQGCRMFALSOGTTLRG VSCDPTGCKMYALHOGTTIRNK VSCSPTECRTFFLTQGSLLNDK YVSCDPDECRFYALSQGTTIRGK FISCSPLECRTFFLTOGALLNDK ISCSPLECRTFFLTOGALLNDK ISCSHLECRTFFLTQGALLND SNGTVKDR SPY ALMSCPLGEAPSPYNSRFESVA LSCPLGEAPSPYNSRFESVI
MSCPLGEAPSPYNSRFESVI

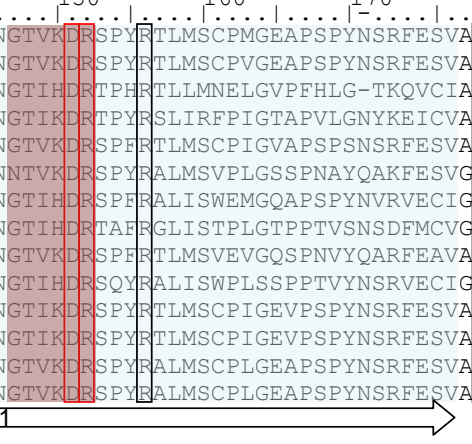

260

270

$\mathrm{dkN} 1 / 05$

$m l N 1 / 09$

$\mathrm{dkN} 2 / 05$

$\mathrm{dkN} \mathbf{3} / 05$

$\mathrm{dkN} \mathbf{4} / 07$

$\mathrm{dkN} 5 / 06$

$\mathrm{dkN} 6 / 04$

dkN7 / 07

$d k \mathbf{N} 8 / 06$

dkN9/ 05

CApdmN1/09

NApdmN $1 / 09$

YAhuN1/06

KIhuN1/06 ................ $220 \quad 230 \quad 240$ GPDNGAVAVLKYNGIITDTIKSWRNDIIRTOE GPDTGAVAVLKYNGIITDTIKSWRNN II RTOES TEC 作 GPDSTAVAVIKYNGIITDTLKSWKGNIMRTO GADDDAYAVI HYGGVPTDVVRSWRKOILRTE SSCVCMKGECYWVMTDGPANNQASYKIFKSQKGLVVDEKEISFQ-GGE GPNNNASAVVWYGGRPVTEI P SWAGN IIR TO E SECVCHKGICPVVMT DGPANNRAATKI IYFKEGKIQKIEELAGN-AQ GPDSKAVAVVHYGGVPTDVVNSWAGDIIRTQESSCTCIQGNCYWVMTDGPANRQAQYRIYKANQGKIVGQTDVSFN-GG GPNNNASAVVWYNRRPVAE INTWARN I I R T E SECVCHNGVCPVVFTDGSATGPADTRVYYFKEGKILKWESLTGT-AK GP DNGAVAVLKYNGIITDTIKSWRNNIIRTQESECACVNGSCFTVMTDGPSNGQASYKIFRIEKGKIVKSVEMNAP-NYHYEECSCYPDSSEITCVCRL GPDGAVAVLKYNG I ITDTIKSWRNN II R TOESECACVNGSCFTVMTDGP SNGQASYKI FRIEKGKIVKSVEMNAP-NYHYE E C CYPDSSEITCVOR GP DNGAVAVLKYNGIITETIKSWKKRIIRTOE SECVCMNGSCFTIMTDGP SNGAASYKIFKIEKGKVTKTIELNAP-NEF GPDNGAVAVLKYNGIITETIKSWKKRILRTESTE

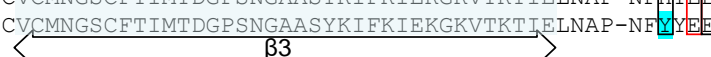
(1)

$$
300 \quad 310
$$

330

340

350 360 370 380 390 WHGSNRPWVSFNO-NLEYQI GYICSGVFGDNPRPNDG--TGSCG-PMSS-NGA-YGVKGESFKYGNGVWIGRTKSTSSRSG EMITDPNGWTETDSSF-S WHGSNRPWVSFNQ-NLEYQIGYICSGVFGDNPRPNDG--TGSCG-PVSS-NGA-YGVKGFSFKYGNGVWIGRTKSTSSR.SGFEMIWDPNGWTETDSSE-S WKGSNRPVIDINMVDYSIDSSYVCSGLVGDTPRNDDSS-SSSNCRDPNNERGN-PGVKGWAFDNGNDVWMGRTISKDSRSGYETFRVIGGWATANSKSQV WKGSNRPWMRINN-ETILETGYVCSKFHSDTPRPADPS-TVS-CDSPSNVNGG-PGVKGFGFKTGNDVWLGRTVSTSGRSGEEIIKVTEGWINSPNHAKS WRGSNRPWIRFNS-DLDYQIGYVCSGVFGDNPRPVDG--TGSCSGPINNGKGR-YGVKGFSFRYGDGVWIGRTKSLESRSGFEMVWDANGWVSTDKDS-N WNGMNRPILTFNE-NLEYEVGYLCAGIPTDTPRVQDSSFTGSCTNAVGGSGTNNYGVKGFGFRQGNSVWAGRTISTSSRSGFEVLLIEDGWIKPSKTI-S WKGANRPVITINPEMMTHTSKYLCSKILTDTSRPNDPI-NGNCDAPITGGSPD-PGVKGFAFLDGENSWLGRTISKDSRSGYEMLKVPNAETDTQSGP-I WOGANRPI IEIDMNTLEHTSRYVCTGILTDTSRPGDKS-SGDCSNPITGSPGA-PGVKGFGFLNGDNTWLGRTISPRSRSGEEMLKIPNAGTDPNSRI-A WTGTNRPVLIISP-DLSYRVGYLCAGLPSDTPRGEDAQFTGSCTSPMGNQG---YGVKGFGFRQGTDVWMGRTISRTSRSSGFEILRVKNGWTQT SKEQ-V WQGSNRPVIQIDPVAMTHTSQYICSPVLTDNPRPNDPN-VGKCNDPYPGNN-N-NGVKGFSYLDGVNTWLGRTISTASRSSGYEMLKVPNALTDDRSKP-I WHGSNRPWVSFNQ-NLEYQIGYICSGIFGDNPRPNDK--TGSCG-PVSS-NGA-NGVKGFSFKYGNGVWIGRTKSISSRNGEEMIWDPNGWTGTDNNE-S WHGSNRPWVSFNO-NLEYQIGYICSGIFGDNPRPNDK--TGSCG-PVSS-NGA-NGVKGFSFKYGNGVWIGRTKSISSRNGEEMIWDPNGWTGTDNNF-S WHGSNRPWVSFNQ-NLDYQIGYICSGVFGDNPRPKDG--EGSCN-PVTV-DGA-DGVKGFSYKYGNGVWIGRTKSNRIRKGFEMIWDPNGWTNTDSDF-S

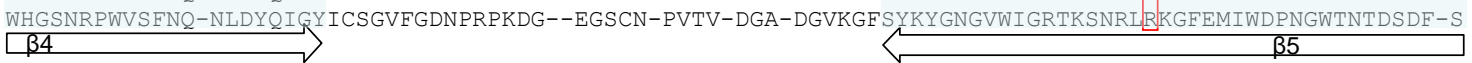

dkN1/ 05

$\mathrm{mlN} 1 / 09$

dkN2/ 05

$\mathrm{dkN} \mathbf{3} / 05$

$\mathrm{dk} \mathbf{N} \mathbf{4} / 07$

$\mathrm{dkN} 5 / 06$

$\mathrm{dkN}$ 6/ 04

$\mathrm{dkN} \mathbf{7} / 07$

$\mathrm{dkN} \mathbf{8} / 06$

dkN9/05

CApdmN1/09

NApdmN $1 / 09$

YAhuN1/ 06

KIhuN1/06

.....400 . . . 410 _.....420

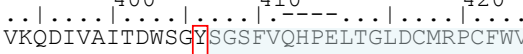
VKQDIVAITDWSGY|SGSFVQHPELTGLDCMRPCFWV DRQVIVDNNNWSGYSGIFS----VEGKSCVNRCFYV ITQTLVSNNDWSGYSGSEI----VENNGCFQPCEYI GVQDI IDNDNWSGYSGSESIRGETTGKNCTVPCFWT KKVEVLSNKNWSGYSGSETIPTAMTSKSCLVPCFWI SHQVIVNNQNWSGYSSGAFIDY-WANK-ECENPCFYY ERQEIVDNNNWSGYSGSFIDY-WNDNSECYNPCEY RKQVVVDNLNWSGY SGSFTLPVELSGKDCLVPCFWV
QGQTIVLNTDWSGYSGSFMDY-WAFG-DCYRACFYV IKQDIVGINEWSGYSGSFVQHPELTGLDCIRPCEWV IKQDIVGINEWSGYSGSFVQHPELTGLDCIRPCFW VKQDIVAITDWSGYSSGSFVQHPELTGLDCIRPCFW $\stackrel{\mathrm{VKQDIVAITDWSG}}{\mathrm{C}}$
$430 \ldots 440$

450 460

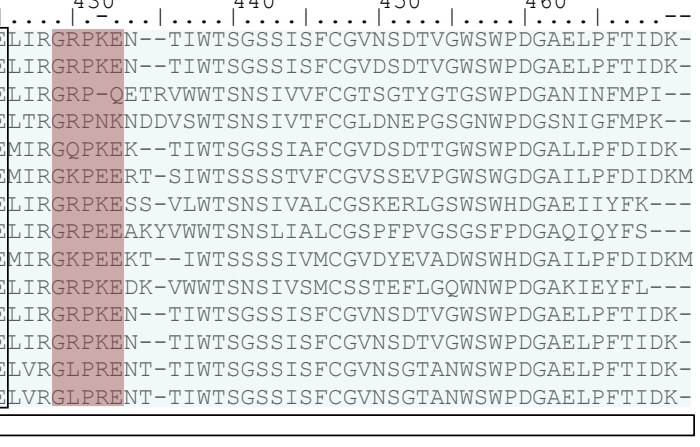


Figure S1. Alignment of (upper panel) huNeus numbered according to each huNeu sequence and (lower panel) viNAs numbered corresponding to the $\mathrm{N} 2$ sequence as shown above the alignment. Residues in transmembrane domains are presented by green texts; ${ }^{1}$ in the case of huNeu 3 and huNeu4, there is no clear evidence which residues are in transmembrane domains. The putative residue at the beginning of the head viNA catalytic cavity is indicated by an arrow. ${ }^{2}$ Highly conserved sequence motifs, REPY/F in viNAs and Y/FRI/VP in huNeus, are highlighted in yellow. The key active-site residues are boxed with red for catalytic site residues and with black for framework site residues. H274Y mutation is indicated by cyan. Residues forming the 150loop and 430-loop in viNAs are highlighted in purple. Three to four aspartic acid box sequence motifs, SXDXGXTW, found in huNeu sequences, but not in the above viNA sequences, are highlighted in gray; however, the biological roles of the Asp boxes remain unclear. ${ }^{3}$ The location forming six blades of the beta-propeller are labeled with light blue and indicated under the sequences. The location forming alpha-helices are highlighted in light green. 


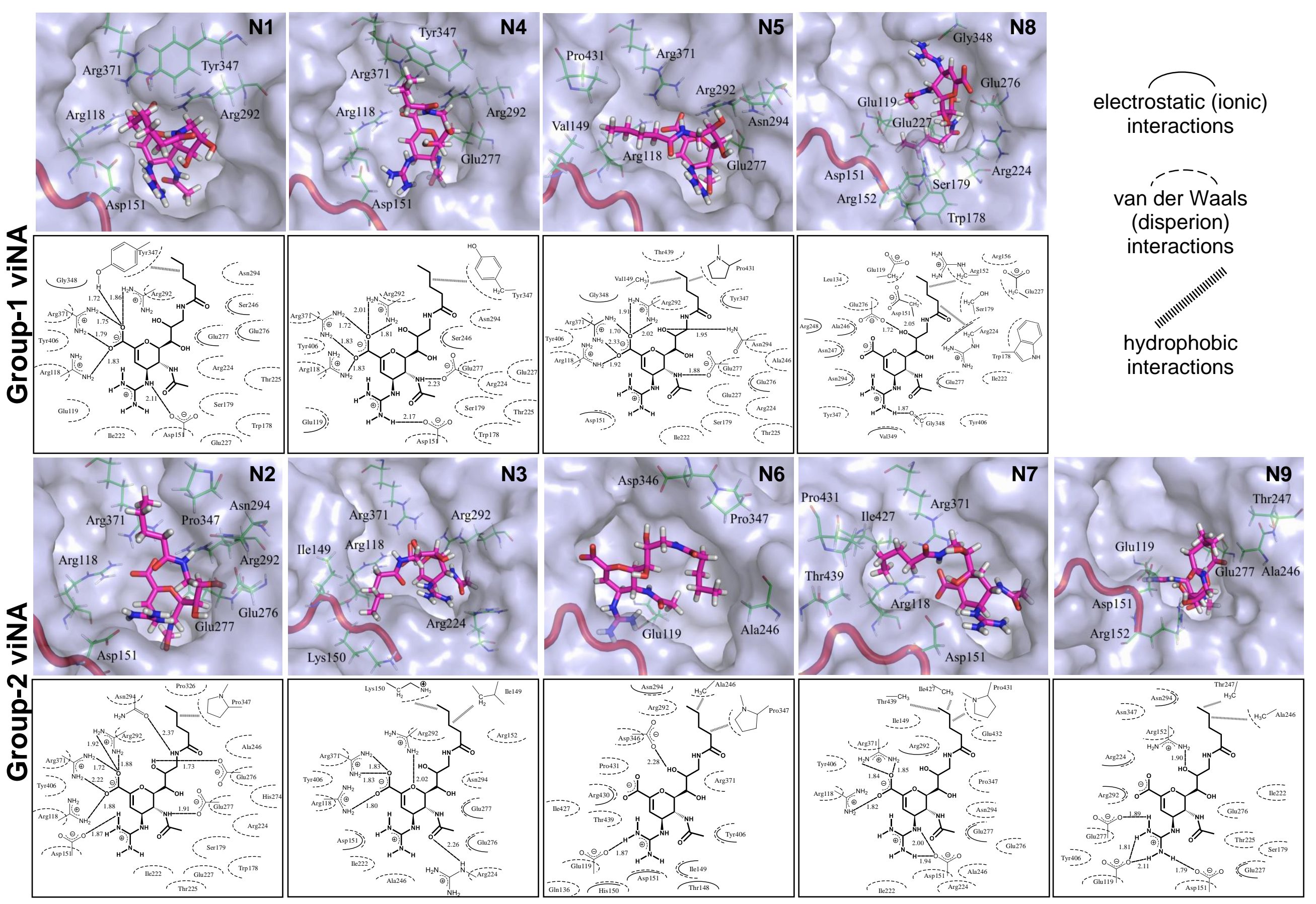

Figure S2. Binding of Pen-GUN in N1-N9 viNA active sites. The Pen-GUN bindings are exhibited in surface diagrams of viNA active sites with the detailed interactions below. 


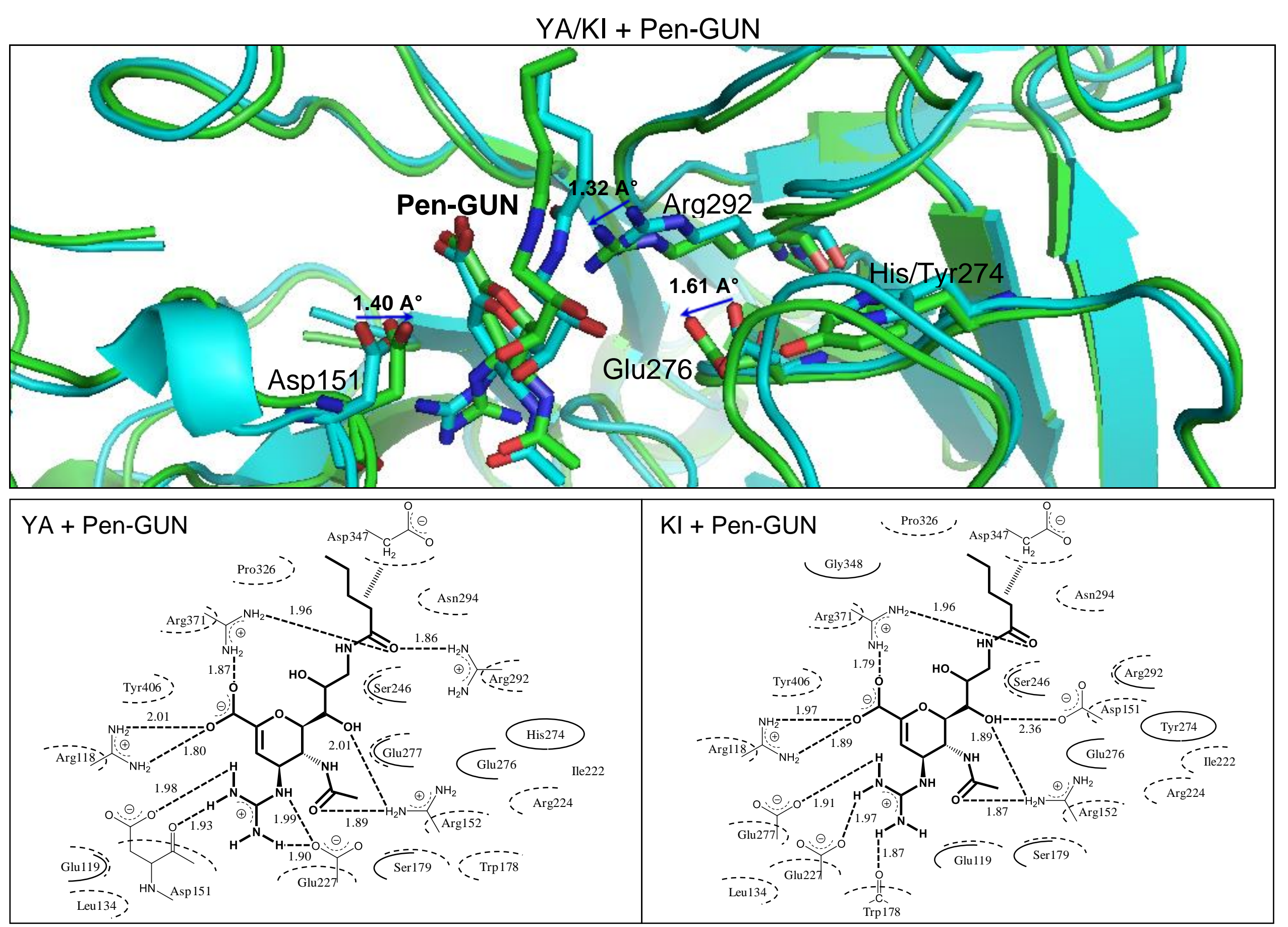

Figure S3. Binding of Pen-GUN in cartoon diagrams of wild-type compared with H274Y-N1 viNA structures. 
Table S1. Comparison of IFIE values of Pen-GUN to cPro-GUN interactions with each important residue in each viNA subtype.

\begin{tabular}{|c|c|c|c|c|c|c|c|c|c|c|c|c|c|c|c|c|c|c|}
\hline \multirow{3}{*}{$\begin{array}{c}\text { PDB model } \\
\text { NA subtype } \\
\text { Residue }\end{array}$} & \multicolumn{2}{|c|}{$3 \mathrm{~B} 7 \mathrm{E}$} & \multicolumn{2}{|c|}{$2 \mathrm{BAT}$} & \multicolumn{2}{|c|}{$2 \mathrm{BAT}$} & \multicolumn{2}{|c|}{ 2HTW } & \multicolumn{2}{|c|}{$3 \mathrm{SAN}$} & \multicolumn{2}{|c|}{$2 \mathrm{CML}$} & \multicolumn{2}{|c|}{ 2QWI } & \multicolumn{2}{|c|}{ 2HTQ } & \multicolumn{2}{|c|}{$2 \mathrm{C} 4 \mathrm{~A}$} \\
\hline & \multicolumn{2}{|c|}{$\mathrm{dkN} 1 / 05$} & \multicolumn{2}{|c|}{$\mathrm{dkN} 2 / 05$} & \multicolumn{2}{|c|}{$\mathrm{dkN} 3 / 05$} & \multicolumn{2}{|c|}{$\mathrm{dkN} 4 / 07$} & \multicolumn{2}{|c|}{$\mathrm{dk} / \mathrm{N} 5 / 06$} & \multicolumn{2}{|c|}{ dkN6/04 } & \multicolumn{2}{|c|}{$\mathrm{dkN7/07}$} & \multicolumn{2}{|c|}{$\mathrm{dkN8/06}$} & \multicolumn{2}{|c|}{ dkN9/05 } \\
\hline & Pen-GUN & cPro-GUN & Pen-GUN & cPro-GUN & Pen-GUN & cPro-GUN & Pen-GUN & cPro-GUN & Pen-GUN & cPro-GUN & Pen-GUN & cPro-GUN & Pen-GUN & cPro-GUN & Pen-GUN & cPro-GUN & Pen-GUN & cPro-GUN \\
\hline R118 & -36.69 & -40.66 & -43.08 & -41.12 & -50.76 & -51.66 & -35.16 & -39.41 & -42.33 & -46.08 & 13.69 & -31.20 & -53.60 & -41.16 & 9.57 & -39.70 & 20.66 & -37.40 \\
\hline E119 & -9.76 & -29.43 & 3.36 & -27.19 & 17.25 & -4.69 & -41.43 & -59.20 & 8.31 & -28.11 & -61.37 & -41.39 & 13.05 & -60.49 & -15.42 & -49.51 & -65.67 & -39.37 \\
\hline L134 & -0.52 & -0.33 & -0.52 & -0.86 & -0.88 & -0.11 & 0.17 & 0.47 & -0.44 & -0.08 & 1.00 & -1.33 & -1.14 & 0.62 & 0.59 & 0.24 & 1.33 & -1.05 \\
\hline Q136 & -1.12 & -1.94 & -0.59 & -1.92 & -1.00 & -2.51 & -1.20 & 0.96 & -0.35 & -3.26 & 1.42 & -2.27 & -1.35 & -1.91 & 0.78 & -1.00 & 0.63 & -2.33 \\
\hline G/N147 & -0.33 & -0.41 & -0.20 & -0.33 & -0.17 & -0.53 & -0.10 & -0.06 & -0.55 & -1.05 & -0.35 & -0.27 & -0.30 & -0.31 & 0.08 & 0.12 & 0.17 & -0.29 \\
\hline V/I149 & -0.24 & -0.85 & 0.44 & -0.68 & -0.51 & -0.35 & -1.41 & -1.82 & 0.30 & -0.89 & -15.19 & -1.15 & 0.49 & -0.53 & -0.48 & -1.39 & -1.54 & -1.28 \\
\hline D151 & -36.83 & -52.48 & -44.27 & -48.57 & -17.65 & -40.71 & -42.14 & -59.14 & -22.85 & -56.41 & -44.17 & -46.19 & -46.57 & 2.26 & -6.48 & -49.91 & -44.17 & -63.16 \\
\hline $\mathrm{R} 152$ & 21.16 & -19.65 & 11.59 & -13.40 & 8.90 & -12.61 & 7.24 & -23.75 & 12.41 & -0.18 & 7.55 & -29.24 & 18.62 & -39.45 & -6.49 & -18.24 & -16.65 & -24.20 \\
\hline W178 & -2.04 & -1.49 & -2.12 & -2.84 & 0.05 & -1.66 & -2.80 & -1.35 & -0.89 & -4.88 & -1.18 & -2.96 & 0.01 & -0.89 & -1.33 & -1.26 & -1.01 & -1.88 \\
\hline $\mathrm{S} 179$ & -5.07 & -26.31 & -3.68 & -31.58 & -0.19 & -16.20 & -5.25 & -3.00 & -3.18 & -34.09 & -2.52 & -31.00 & -0.31 & -6.69 & -4.78 & -13.01 & -4.26 & -30.87 \\
\hline $\mathrm{I} 222$ & -2.04 & -3.62 & -3.68 & -7.45 & -2.32 & -2.85 & -0.79 & -1.59 & -3.12 & -0.42 & -0.35 & -1.48 & -1.66 & -1.44 & -1.15 & -3.75 & -1.60 & -1.09 \\
\hline R224 & 7.99 & 3.04 & 12.97 & 8.35 & 0.83 & 20.85 & 4.36 & 2.40 & 7.40 & 4.15 & 3.96 & 8.15 & 7.92 & 10.05 & -11.06 & 8.63 & -12.39 & -1.92 \\
\hline E227 & -12.75 & -45.41 & -8.41 & -40.61 & 1.87 & -61.67 & -17.76 & -12.34 & -6.71 & -45.48 & -12.52 & -35.67 & 2.83 & -80.39 & -14.71 & -26.00 & -15.41 & -27.61 \\
\hline $\mathrm{T} 242$ & 1.02 & 1.16 & 0.14 & 0.54 & -0.70 & -0.03 & 0.94 & 0.71 & 0.57 & 0.98 & 0.52 & 1.97 & 0.47 & 1.78 & -0.33 & 0.85 & 0.03 & 1.13 \\
\hline S/A246 & -7.07 & -4.76 & -3.55 & -4.55 & -3.76 & -0.88 & -6.22 & -5.05 & -1.87 & -0.91 & -0.72 & -3.94 & -2.08 & -2.73 & -9.69 & -1.01 & -4.66 & -2.76 \\
\hline E276 & -12.93 & 4.48 & -45.91 & -46.91 & -18.00 & -29.03 & -7.68 & 3.13 & -23.66 & -0.68 & -5.15 & -20.05 & -13.96 & -12.49 & -52.02 & -4.82 & 0.88 & -17.37 \\
\hline E277 & -17.60 & -14.87 & -18.03 & -23.21 & -3.93 & -48.25 & -19.60 & -3.87 & -17.44 & -32.35 & -14.55 & -22.72 & -2.12 & -48.84 & -22.19 & -0.49 & -40.52 & -11.80 \\
\hline R292 & -37.77 & -55.26 & -51.38 & -41.11 & -25.53 & -16.67 & -49.04 & -37.49 & -45.75 & -52.08 & 4.42 & -38.21 & -9.19 & -13.05 & 24.34 & -45.92 & -25.34 & -53.31 \\
\hline N294 & 0.37 & 7.82 & -3.31 & 4.12 & 2.32 & -4.68 & 4.71 & -0.22 & -0.28 & 9.53 & -4.93 & -5.82 & -3.68 & 1.48 & -10.84 & 6.27 & -11.37 & 5.58 \\
\hline N/T325 & 1.00 & 1.03 & -0.98 & -1.10 & -1.06 & -0.87 & 0.10 & 0.17 & -0.74 & -1.47 & 0.77 & -1.15 & 0.24 & 0.67 & 1.69 & -1.48 & -1.09 & 0.71 \\
\hline P/S326 & 2.09 & 3.02 & 1.50 & 1.98 & 1.85 & 1.48 & 1.87 & 1.99 & 1.75 & 2.79 & -0.95 & 2.91 & 0.17 & -0.36 & -4.15 & 2.78 & 0.22 & 2.76 \\
\hline Y/P/N347 & -19.11 & -20.95 & -1.60 & -0.45 & -0.08 & -1.98 & -9.73 & -8.33 & -11.22 & 1.10 & -3.30 & -4.23 & -3.91 & -8.06 & -7.40 & -22.65 & -3.01 & 6.22 \\
\hline G348 & -2.43 & -3.54 & -1.61 & -2.18 & -0.47 & -2.35 & -0.61 & -1.30 & -1.69 & -4.84 & -1.06 & -3.35 & 1.07 & -0.68 & 1.23 & -3.14 & 1.00 & -4.06 \\
\hline V349 & 10.03 & 12.66 & 6.75 & 9.22 & 7.79 & 6.24 & 9.60 & 9.47 & 8.72 & 12.40 & -6.42 & 7.98 & 2.61 & 9.16 & -32.46 & 11.70 & -2.60 & 11.55 \\
\hline S/G370 & 0.72 & 1.06 & 0.75 & 1.45 & 0.03 & 0.74 & 0.59 & 0.67 & 0.12 & 0.74 & 1.39 & 0.39 & 0.22 & 0.72 & -2.10 & 1.68 & -0.07 & 0.76 \\
\hline R371 & -83.97 & -98.19 & -88.65 & -98.18 & -77.44 & -94.97 & -82.15 & -84.49 & -76.77 & -98.22 & -2.63 & -82.30 & -64.40 & -88.30 & 23.39 & -84.53 & 4.84 & -94.17 \\
\hline Y406 & 1.73 & 6.98 & 3.65 & 5.92 & 0.35 & 5.56 & 3.68 & 4.48 & 3.50 & 0.59 & -5.12 & 5.16 & -1.70 & 2.63 & -5.66 & 3.90 & 4.27 & 2.94 \\
\hline $\mathrm{I} / \mathrm{T} 427$ & 0.00 & 0.03 & -0.65 & -0.75 & -0.71 & -0.33 & -0.08 & -0.01 & -0.27 & -0.44 & -1.71 & -0.12 & -1.00 & 0.18 & 0.28 & -0.50 & 0.00 & -0.14 \\
\hline P431 & 0.81 & 0.84 & 0.74 & 0.52 & 0.63 & -0.54 & 0.14 & -0.13 & -0.23 & -2.08 & -7.68 & 0.06 & -0.98 & 0.40 & -0.51 & -5.13 & -0.96 & 0.23 \\
\hline K/Q/N/E432 & -11.12 & -15.85 & 0.25 & 0.09 & -0.26 & -0.88 & -6.90 & -7.79 & 6.86 & 17.10 & -20.47 & -14.07 & 6.22 & 12.20 & -5.22 & 9.26 & 2.38 & -8.73 \\
\hline T439 & 0.14 & 0.62 & -0.07 & 0.68 & -0.19 & 0.94 & 0.25 & 0.28 & -0.19 & 0.66 & 1.28 & 0.78 & -0.85 & 0.32 & 0.09 & -0.54 & 0.38 & 0.68 \\
\hline
\end{tabular}




\section{References}

(1) Barman, S.; Nayak, D. P. Analysis of the transmembrane domain of influenza virus neuraminidase, a type II transmembrane glycoprotein, for apical sorting and raft association. J. Virol. 2000, 74, 6538-6545.

(2) Ward, C. W.; Elleman, T. C.; Azad, A. A. Amino acid sequence of the Pronase-released heads of neuraminidase subtype N2 from the Asian strain A/Tokyo/3/67 of influenza virus. Biochem. J. 1982, 207, 91-95.

(3) Chavas, L. M.; Tringali, C.; Fusi, P.; Venerando, B.; Tettamanti, G.; Kato, R.; Monti, E.; Wakatsuki, S. Crystal structure of the human cytosolic sialidase Neu2. Evidence for the dynamic nature of substrate recognition. J. Biol. Chem. 2005, 280, 469-475. 